\title{
Crowdsourcing 3D Cultural Heritage: Best Practice for Mass Photogrammetry
}

\author{
Eugene Ch'ng, NVIDIA Joint-Lab on Mixed Reality, University of Nottingham Ningbo China \\ Evelyn Tong Zhang and Shengdan Cai, NVIDIA Joint-Lab on Mixed Reality, University of Nottingham \\ Ningbo China \\ Fui-Theng Leow, Faculty of Business, University of Nottingham Ningbo China
}

\begin{abstract}
Purpose - The paper presents the rationale for democratising the digital reproduction of cultural heritage via 'mass photogrammetry', by providing approaches to digitise objects from cultural heritage collections housed in museums or private spaces using devices and photogrammetry techniques accessible to the public. The article is intended as a democratised approach rather than as a 'scientific approach' for the purpose that mass photogrammetry can be achieved at scale.

Design/methodology/approach - The methodology aims to turn the art of photogrammetry into a more mechanical approach by overcoming common difficulties faced within exhibition spaces. This approach is replicable and allows anyone possessing inexpensive equipment with basic knowledge of photogrammetry to achieve acceptable results.

Findings - We present our experience of acquiring over 300 3D models through photogrammetry from over 25 priority sites and museums in East Asia. Our approach covers the entire process from capturing to editing, and importing 3D models into integrated development environments for displays such as interactive 3D, Virtual Reality (VR) and Augmented Reality (AR).

Practical implications - The simplistic approach for democratised, mass photogrammetry has implications for stirring public interests in the digital preservation of heritage objects in countries where museums and cultural institutions have little access to digital teams, provided that Intellectual Property (IP) issues are cared for. The approach to mass photogrammetry also means that personal cultural heritage objects hidden within the homes of various societies, and relics in circulation in the antiques market can be made accessible globally at scale.
\end{abstract}

Originality/value - This article focuses on the complete practical nature of photogrammetry conducted within cultural institutions. We provide a means for the public to conduct good 
photogrammetry so that all cultural heritage objects can be digitally recorded and shared globally so as to promote cross-cultural appreciation of material cultures from the past.

Keywords: photogrammetry, digital museum, crowdsourcing, best practices, VR, AR

Paper type: Technical paper

\section{Introduction}

The significant change in the accessibility of software in the last decade has inadvertently paved the way for the 'now everyone can do it!' paradigm (Ch'ng et al., 2014). This datahungry generation has participated in the creation and sharing of digital images and videos across the Web and social media. This sharing ecology can be attributed to the convenience of present day mobile Apps and the sharing framework provided by social media services. In our photogrammetry activities and in our witnessing of the digital needs of museums, which have culminated in the preparation of this article, we felt that cultural heritage objects will receive the greatest attention in the future of photogrammetry. This, we feel, can be ascribed to the creator and the sharer's intrigue towards a cultural object's importance to his or her national identity or personal history as a priority, and the source of inspiration for creative works in the creative economy as the second most important priority.

Driven by recent technological advances in Smartphones embedding high resolution digital cameras, the computational power of GPUs (graphical processing units), along with distributed processing algorithms, photogrammetry is now a low cost, accessible technology for producing photorealistic three-dimensional digital models of objects, monuments, sites, designs and artworks. The general belief in conducting good photogrammetry work appears to focus on the software and equipment possessed by a professional team. While these are central to the high quality, more 'scientific' approach to the reproduction of facsimiles of relics, the actual work itself requires manual labour and skill, and the suitability of the environment housing the object. In addition, it is also the need for human labour that prompted us to consider the need for such an article. Our argument is that good photogrammetry involves much more than algorithms, which past literatures (Luhmann et al., 2006) are focused on. At the time of writing, guidance on the other aspects of work involving human input specific to the context of museums were not found. The focus of our paper is this 'other aspect' of photogrammetry work.

This paper serves to provide a best practices guide to mass photogrammetry with the aim of stirring public interests for crowdsourced heritage contents. While issues and implications of IP and copyright raised as a result of photogrammetry work are beyond the scope of this paper, we debated these matters in another article presently in the publication pipeline. Rather, we focus on the practical issues of photogrammetry work, examining the most efficient means of producing quality 3D representational objects from the confines of, and the 
controlled environment in which museums and the general GLAM (galleries, libraries, archives, and museums) institutions were built upon. We believe that whilst high-quality equipment and specialists skills are needed for high quality digital preservation work, not many cultural institutions possess the resources and a professional team needed to carry out photogrammetry on a massive scale. Besides, due to the cost of it, digital teams are commissioned with a specific focus, on special collections, and that many cultural heritage objects including monuments are deemed too unimportant by curators for digitisation. The costs measured against the value of professionally digitising large quantities of objects are often unjustifiable. As a consequence, the majority of objects are not digitised, and are unlikely to be publicly accessible across the expanse of time and space. This necessarily leaves the job to crowdsourcing work, if only the population can be mobilised and empowered with practical knowledge and institutional-level encouragement.

We feel that accessibility via digital means is a necessary transformative practise in the $21^{\text {st }}$ century, for "unrestricted access to the archives provides two major benefits. The first advantage allows the rediscovery of hidden source of information that may bridge relationship or chronological gaps amongst objects. This helps extend current knowledge for researchers." (Ch'ng, 2013). Mass photogrammetry exercise is not nugatory. The value for research, cultural exchange, and heritage as a source of creativity that can be generated as a result of crowdsourced digitisation exercises can be extensive. Multiple collections hosted across global museums of different levels, objects in circulation in the antique market, and heritage inherited in private collections can be made globally accessible so that 'rediscovery of hidden source of information that may bridge relationship or chronological gaps amongst object' (ibid.) may be achieved. In the course of value creation as a result of mass photogrammetry activities, an important question to ask is the need for a 'scientific approach' in the digitisation of objects - must digitised objects be documented in full surface quality, or is a capture of the likeness of the object sufficient? The answer to such a question depended on the use-value of the target object. If the purpose of documentation is for digital preservation and professional and scholarly inspection, a full quality capture equivalent to a professional laser-scan is mandatory. This produces a true facsimile of the object. But for public consumption, cultural exchange, creative inspiration and even research, where surveys of digital objects and exploratory searches of digital artefacts may yield interesting discourse, an adequate reproduction in the likeness of the original physical object may be sufficient. Any identified object of interests may then be digitised as necessary later by professional commission. In fact, no professional digital team would share full quality models on the Web by reason of effort, copyright and ownership. Furthermore, 3D object sharing platforms such as SketchFab require that models be heavily decimated. A 'scientific approach' to professional photogrammetry as compared to a 'simplistic approach' used in mass photogrammetry has very different nature and utility.

Our study has produced over 300 objects classed as national treasures within top-tiered museums in East Asia, which could be used, if care is taken, in derivative works related to digital heritage, or creative works in art and design and even 3D prints. We recommend that 
any such objects digitised from museums provide the objects' affiliations, by practicing good etiquettes in linking the objects to the museums so that the cultural heritage institutions are credited. The ReACH programme (Reproduction of Art and Cultural Heritage) guidelines should be consulted (ReACHDeclaration, 2018) so that the reproduction of objects via digital means can become sustainably useful.

We begin with a literature review in the next section, briefly covering photogrammetry and its implications for the digital reproduction, storage and sharing of cultural heritage. The literature also posits photogrammetry within the context of the democratisation of technology, and as a means for crowdsourcing digital assets in cases where cultural institutions lacked financial and human resources for digitisation work. The literature review is concluded with the implications of IP issues if mass photogrammetry is practiced. The article then continues with the methods section (section 3), presenting and comparing the outputs in section 4 . The article finally concludes with a discussion of the impacts of mass photogrammetry for both society and cultural institutions.

\section{Literature Review}

\subsection{Photogrammetry - from the Professionals to the Masses}

Photogrammetry is known as an image-based systematic method for measuring and recovering the positions of surface points from photographic features for the purpose of producing 3D digital copies from physical objects. An important text for close-range photogrammetry, its principles, techniques and applications will provide the necessary technical foundations for our readership (Luhmann et al., 2006). In this article, we extended the work to a specific context requiring crowdsourcing work at scale.

Photogrammetry is a more flexible approach for data acquisition, resulting in objects with a higher level of details, accuracy and range (Hanan et al., 2015; Samaan et al., 2016). It is becoming a common method for creating 3D virtual environments (Koller and Levoy, 2004; Swearingen and Swearingen, 2016; Cai, Ch'ng and Li, 2018; Li et al., 2018). There is a large body of literatures on the technicality of 3D capture, which is outside the boundary of the focus of this article. For brevity, articles reviewing the techniques and applications of 3D imaging are provided here for reference (Rocchini et al., 2001; Böhler and Marbs, 2004; Yastikli, 2007; Sansoni, Trebeschi and Docchio, 2009; Remondino, 2011). Photogrammetry techniques and technologies are being developed, and will be of greater utility with integrated hardware, see for example, Cultlab3D's solution for making photogrammetry work easier (http://www.cultlab3d.de), and techniques for automating the decimation of models (https://blog.sketchfab.com/tutorial-low-poly-assets-from-3d-scans/) amongst others.

Increased access to a combination of digital and modular technologies are fueling an explosion of hobbyist within the hacker-space and maker movement, which has positively transformed "the dominant paradigm of user-as-consumer" to "alternative framings of the user as creative appropriator, hacker, tinkerer, artist, and even co-designer or coengineer"'(Tanenbaum et al., 2013). In parallel to these movements, smartphones and the 
availability of powerful desktops or photogrammetry Cloud services are allowing an increasing number of non-specialists with interests in digitisation works to produce 3D models from photographs. This phenomenon was first mentioned as 'mass photogrammetry' (Granshaw, 2015). The 3D models generated by mass photogrammetry can then be used for derivative works such as interactive websites, mobile Apps, touchtables (see Ch'ng, 2013 for details on digitised fossils of Trilobites becoming alive) and 3D printing (Hanan et al., 2015; Miles et al., 2016; Samaan et al., 2016).

Although case studies of good photogrammetry work exists, an example is the reconstruction of the traditional Batak Toba house and its natural environment (Hanan et al., 2015). Others such as Cai et al. (Cai, Ch'ng and Li, 2018), uses photogrammetry in VR for testing cues for memory and nostalgia, and Li. et. al. (Li et al., 2018) made use of photogrammetry for a hybrid VR-AR environment. For the most part, works related to photogrammetry either focuses on the computational aspects of it, or the application of photogrammetry to other works; there was nothing in between on methods and best practices. Therefore, a good guide in the manual aspects of photogrammetry has become necessary. Such a guide promises potentials for using the data for research and creative works for individuals, for labs and start-ups with limited resources, and for communities interested in reproducing artefacts with nationalistic, symbolic or personal value. In this paper, we demonstrate that photogrammetry can be a democratised technology, with best practices structured from our studies applied in the museum contexts. Our article covers the challenges and potentials arisen as a result of photogrammetry technology. An understanding of good photogrammetry work leading to the mass reproduction of models can empower creative primaries and derivative works.

We also regard mass photogrammetry as an important activity to heritage at risk from terrorist destruction, natural calamities, rapid economic development, and anthropogenic hazards. Our readership may wish to refer to the AHRC (Arts and Humanities Research Council) funded Curious Travellers' project for more details on our crowdsourced infrastructure to help with the digital documentation of archaeological sites, monuments and heritage at risk (www.visualisingheritage.org).

\subsection{Use of Mass Photogrammetry for Cultural Heritage - IP \& Copyright Issues and Crowdsourcing Potential}

The cultural heritage of nations are assets from which social, cultural, and economic potentials may be realised (Greffe, 2004). A nation is formed by its culture in which its shared memory from a common past builds the sense of community (Anderson, 2006). Hence, a nation's cultural heritage plays an important role in generating and maintaining the citizens' national identity. This connotation of what a nation is, and how it is linked to heritage makes it important to preserve recorded heritage and personal heritage, such as objects passed down from past generations in private collections. In the creative industry, heritage is a source of creativity and monetary profit. It provides knowledge of historic 
experiences and draws its unique identity, inspiring inventions and innovation within a society (Potts and Cunningham, 2008), (Beyond Productivity: Information Technology, Innovation and Creativity, 2003). There is a good reason why the combined keyword "digitisation and preservation" has become important in contemporary society. The UNESCO's 'The Memory of the World in the Digital Age: Digitization and Preservation' held in Vancouver, Canada in 2012 to mark the 20th anniversary of the UNESCO programme manifests an important juncture towards the 'repositioning and strengthening [of] the information profession to play a key role in global development' (Thurston, 2012). The setting up of the national committee of ICOMOS (Hughes, 2015) to address the use of 'Big Data' and 'Digital Technologies' in culture and cultural heritage is a further testament of the importance of using digital technologies for the preservation and communication of heritage.

Tourists tended to take photos within museums and heritage sites, and posting them to social media. This inadvertently leads to a rich collection of photos of multiple angles of objects, from which 3D objects may be generated. However, if visitors know the possibility and ease of reconstructing 3D models of heritage objects, they may not hesitate in doing so, the consequent being that 'digital thievery' is practiced. The likelihood of the use of mobile phones for controversial purposes has been mentioned before (Nightingale, 2007), for "access to cheap flexible tools removes many of the barriers to trying new things" (Shirky, 2010). Whatever the reason, for mischief or for creativity, cultural heritage as a nation's identity must have some sort of IP protection. On the positive side, museums can be avenues of inspiration for creative works (see Sabiescu et al., 2015 and Younan, 2015). However, issues may arise without proper authorisation and the association of the right information to 3D models of cultural heritage produced by amateurs.

Various arguments related to IP rights dedicated to museums and cultural heritage contents have been debated (Pantalony, 2006; Derclaye, 2010; Margoni, 2014; Project, 2016), these focus on 2D images, 3D models are rarely mentioned. Our article extends this matter from 2D to $3 \mathrm{D}$, by demonstrating the ease of photogrammetry with highly accessible tools which amateur could acquire, and argue in favour of the adoption of digital IP management in museums. Museums should digitise their own contents so as to benefit themselves, their consumers, and creators from the protection afforded by appropriate IP rights. In terms of museum practices, very few provides online 3D objects of their collections. The Metropolitan Museum of Art in New York first invited technology and heritage enthusiasts in 2012 to digitise models so that they can be shared, downloaded and adapted to create new creative works, and for commercial works (see https://www.thingiverse.com/met/about). The case is a success in mobilising the community to carry out digitisation works, and also illustrated the feasibility and the potentials of launching large-scale online crowdsourcing to meet larger demands.

Crowdsourcing projects have become pervasive in the cultural heritage domain. For example, the photo-tagging project on the Vele Handen (Noordegraaf et al., 2014), global search for stolen antiques (TNN, 2017), historic newspaper correction project by the Cambridge Public library (Zarndt et al., 2013), and especially those which assist in the digital 
preservation of heritage threatened by widespread terrorist destruction, such as the Curious Traveller's project (www.visualisingheritage.org) and UNESCO's ReclaimHistory.org. Similarly, British Museum has released 242 pieces of 3D models since 2014 in collaboration with Sketchfab (https://sketchfab.com/britishmuseum), an online platform in which users view, share and download models for non-commercial purposes. In the case of the British Museum, only 242 models were made available, at the third year since the venture. With 8 million objects in the collection, this could take thousands of years. This reflects the large financial, technical, and human resources needed for digitisation work. In January of 2018, the 242 models have attracted 7,105,000 views, with the most viewed model being the Jericho Skull. The skull was downloaded over 2.5 million times, demonstrating the popularity of $3 \mathrm{D}$ objects with the public, if only the public could take part in the work. At the ReACH technical policy roundtable discussions held at the Department of Culture and Tourism, Abu Dhabi UAE (23-24 April 2018), directors of large, established museums communicated the difficulty of finances needing to digitally record and store artefacts. This quiet protest reveals the greater, global outcry among small cultural institutions and groups with limited resources.

\section{Methods}

Crowdsourced digitisation of cultural heritage artefacts can be achieved via photogrammetry. Where target objects are in your possession, photogrammetry techniques can be achieved quite effectively. However, in specific situations where your subject is affixed in a location, and encased within glass containers with artificial lightings fixed at certain angles with lights cast on surfaces of the object, photogrammetry can become challenging. This is why a best practices guide accompanying the rationale for mass photogrammetry can become useful. Here we present thirteen subsections where the challenges frequently faced by photogrammetry users within public spaces are discussed in detailed. We wish to note that this article is presented as a guide, and that the results of photogrammetry do depend on many factors such as the environment of the museum, the camera used, the algorithms performed on the image sequence, the material of the object, and etc. Whilst the visual similarity of the model is discernible from the original object, the results would be difficult to quantify due to the many factors involved. However, the likeness and detail of the produced models can be visually evaluated by users. As this is a paper encouraging 'mass photogrammetry', with perhaps $80-90 \%$ of citizens not having access to professional equipment, we will not delve into the quality of the 3D geometry which are often judged by professional photogrammetrists. Furthermore, we will not elaborate the computational process of photogrammetry as it is well known and can be read from various literatures. Instead, we felt that a formulated guide is a necessary complement for generating good quality models. Here, we provide an initial descriptive formula, a model for good photogrammetry work. Based on our study of over 300 heritage objects, our understanding is that the quality of the model produced by any photogrammetric process is a function of individually weighted variables, such as the object's material and form $\mu$, the object's environment $\varepsilon$, the devices used $\delta$, and the skill of the photographer $\zeta$ including standard 
practice: $M=w_{1} \mu+w_{2} \varepsilon+w_{3} \delta+w_{4} \zeta$ where $\mathrm{M}$ is in the range [0,1] with the arbitrary weights indicating how much a person have control over a given variable. The four variables within the function can be controlled whereas the automated computation of the software is fixed. The goal is to increase the weights of all the variables and thereby produce the best output. For museum environments, $w_{1}$ and $w_{2}$ are largely fixed, which makes it highly challenging. Our article contributes in optimising the weights of all four variables.

\subsection{Software and Hardware}

Photogrammetry software systems are available as Cloud services and stand-alone software. VisualSFM (Structure From Motion) (Wu, 2011) is a software for processing 3D models using image features mapped from different viewpoints around an object of interest. Other proprietary software are Agisoft PhotoScan, a software that processes digital images and generates 3D spatial data used in GIS applications and object digitisation. The PhotoModeler is another package with similar features, used for surveying, mapping and measurements. AutoDesk ReCap and especially Reality Capture are popular systems in use by professional groups but the licenses are more expensive. We are aware that there will be many more such software made available in the immediate future where the public can capitalise on.

For our case suited to mass photogrammetry, and for ease of use and outputs in AR and VR applications, we opted for VisualFSM and especially Autodesk ReCap, a standalone software and Cloud photogrammetry service that has ease of editing, transferable models between Autodesk software and export features for VR and AR environments such as Unity and Unreal Engine. Our RealityCapture licenses are used for more professional preservation works. We also used a series of NVIDIA-based hardware, including the workstations that come with 32/64GBs of RAM and house the NVIDIA GPUs we used: Quadro M6000s, Quadro K4000s, GTX1080s, for processing the images locally in VisualSFM.

\subsection{Photogrammetry Devices}

We tested three devices in order to compare the quality of models:

- Canon 6D with Canon Zoom Lens EF 24-70mm 1:4L IS USM at

- Samsung S7 with HDR

- Apple iPhone 7 Plus with HDR

We made use of affordable smartphones for the majority of our work but compared the three cameras for signs of defects and quality. The models produced from the deliberate use of our low cost equipment affordable to the public are presented in section 4 . 


\subsection{Choosing a Site}

We selected East Asia's top-tiered museums due to the richness, complexity and quality of the curated objects and their significance to a nation's rich history and identity. These sites were located mainly in China, including Taiwan and Hong Kong, Japan, Thailand, Malaysia and Singapore. The choice we have made presented us with various difficulties in terms of visitor crowding, inconsistent background, lighting conditions, locations of objects of interest and their display casings. These challenges naturally become factors from which we were able to realise the ambitions of good mass photogrammetry work.

\subsection{What to Wear}

Wearing appropriately coloured clothing can be important. The glass casing of displays is often reflective. While the colour of the clothing of other visitors are not important mainly due to their tendency to keep a distance from other viewers, your proximity to the casing during photogrammetry work can be affected by your own clothing. Dark coloured clothes with long sleeves and non-glossy ornaments such as buttons, rings, necklaces, bracelets or watches are recommended. Glossy objects reflected on glass casing are known to create defects on the model.

\subsection{Spaces and Displays}

The general rule for photogrammetry work is that sufficient space is needed for manoeuvring your camera $360^{\circ}$ around the display to construct a complete object. If an object is displayed in front of a wall, you may only capture at most a $270^{\circ}$ series of images and, as a consequent, only a partial 3D object is produced (Figure 1A). Insufficient lighting or shadows may contribute to missing polygons (Figure 1B). However, the unspoken rule in most museums, particularly museums within China are to have the most valuable object displayed within a glass case with ample spaces around for visitor admiration. Therefore, national treasures of significance naturally make it easier for crowdsourcing 3D models from.

Objects are almost always in glass cases as a preventive measure. This is an issue when spotlights are strong. Spotlights from other displays can cast reflections on the glass casing of the target object, which could reduce the quality of the generated model, such as blurring and distorting objects (Figure 1C). Polarising filters may be used for reducing reflections; they are available for all types of cameras and smartphones.

\subsection{Lighting Conditions}

Various lighting conditions such as the evenness, intensity and temperature are all important factors in generating 3D models that can represent the subjects. Objects placed outdoors have preferable lighting conditions, as ambient sunlight is more even at certain time of day and are generally brighter. The general rule is mild, even lighting conditions casts on all surfaces of the object are better. Strong, direct sunlight will produce models with shadows 
overlapping the texture of the object. The best times for outdoor photogrammetry work are when light is even, i.e., when the sky is overcast and cloudy but with strong sunlight behind the clouds. Strong light and shadows can be normalised if RAW images are captured. RAW image files can be produced by most digital SLR cameras and some smartphones.

\subsection{The Nature of an Object's Material}

Photogrammetry requires feature matching between photographs, as such the material of objects influences the quality of models. Similar to 3D laser scanning, transparent and translucent objects (Figure 1D) are almost impossible to reconstruct unless a coat of powder is applied. This is intrusive and permission is unlikely to be given from the host museums. Figure 1D shows a failed transparent object. Glossy surfaces could be possible although defects are usually found on 3D models after production (Figure 1E) even if lighting conditions are appropriate. However, minor defects are not difficult to fix using the editor. The ability to digitise the shape of glossy objects makes photogrammetry superior to laser scans. In general objects with matt surfaces, which do not reflect light are perfect for photogrammetry work. Objects that have rough surfaces are suitable for photogrammetry work but additional close-up photography is needed to capture the details. We have also found that coloured objects with rich features are easier to digitise than objects with smooth surfaces. This can be attributed to the ability of photogrammetry algorithms to conduct feature mapping from the photographs.

\subsection{Bases and Backgrounds of Objects}

The background where an object is positioned is crucial for successful feature mapping in photogrammetry work. Backgrounds and bases (e.g., pedestals) that are not strongly contrasted with the foreground object can post an issue, as digitised models will be fused with the base of the object (Figure 1F). However, museum displays seldom have non-contrasted bases as the prominence of the objects is a priority. We suggest that an extra cycle of close-up photographs be taken of the base and the top for better 3D reconstruction. Photogrammetry activities require that the photographer walks around the object, taking shots at measured angles. Therefore, the background of the object changes at every angle. The presence of passing visitors in the background could post problems as this may confuse automated feature mapping within photogrammetry software. However, we have not had any issues so far. 


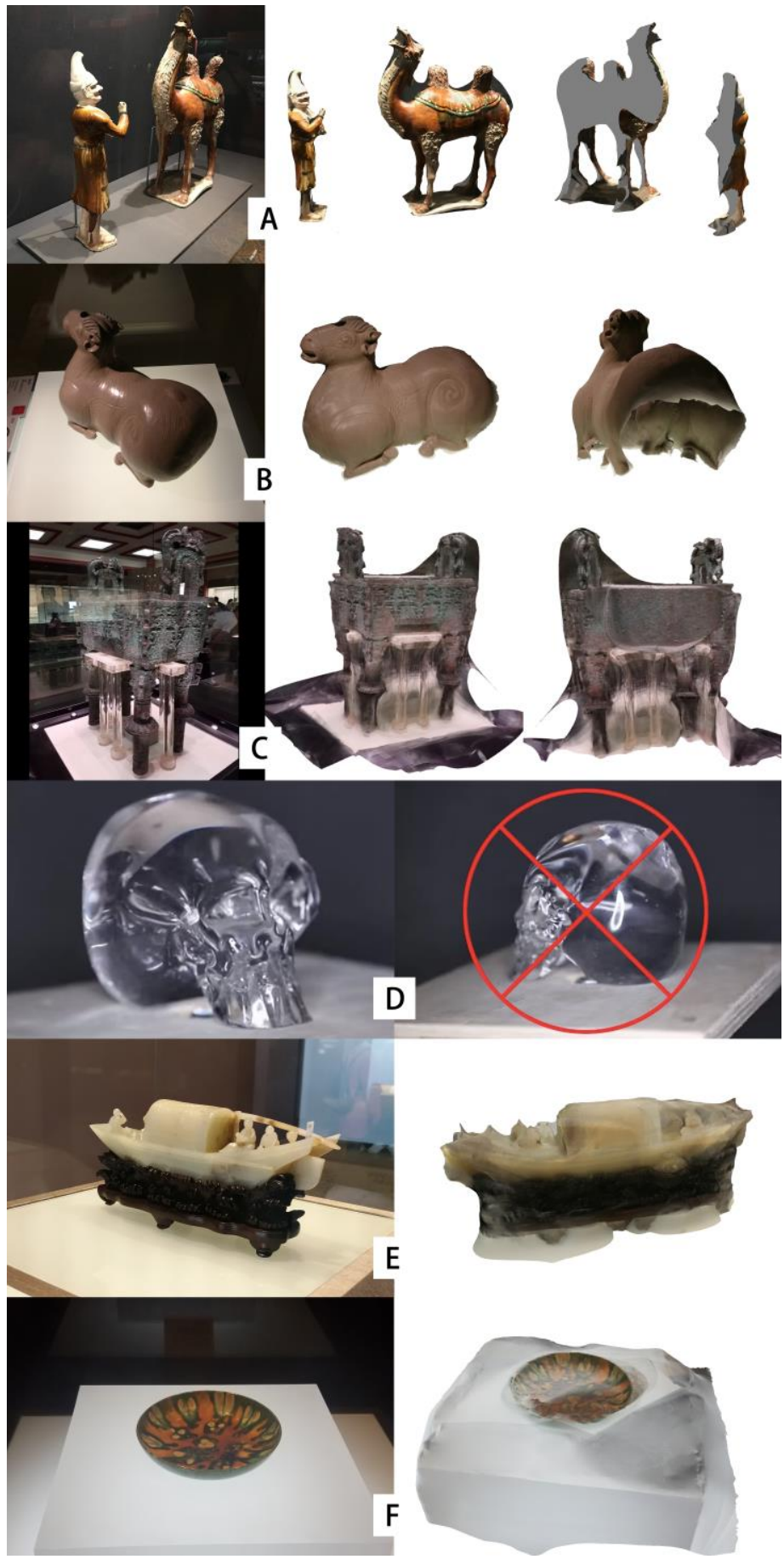

Fig. 1. Defective objects caused by challenging environments unsuited for photogrammetry work. 


\subsection{Object Metadata and Paradata}

3D objects acquired from photogrammetry must be identifiable for it to have value of any kind. The series of photographs taken may not necessarily contain the object's information. It is advisable to take additional photographs of an object's captions so as to identify it later. Objects can be unidentifiable in the confusion of hundreds of photogrammetry images, we recommend naming your folders properly where each set of images representing the objects are respectively stored. Note that many museums do not publish their object metadata via online channels, and therefore you are required to acquire as much information as possible onsite, particularly if the site is challenging geographically. Your device should also automatically capture photographic settings and geo-tagged locations (if switched on). These are embedded within the digital images as metadata, which can be obtained via image processing software. The paradata, the process by which the photogrammetry was conducted, including the person carrying out the work, the duration, time of day, equipment used, etc should also be recorded. The idea is to record as much information as possible, for the object to be of value to future audiences and consumers. The ReACH Technical Policy guideline can be consulted for the record of metadata and paradata.

\subsection{Capturing Image Sequences}

There are various approaches for manoeuvring your photographic device around an object. Video-recording an object is an approach. Alternated sequence from photographs can be extracted from videos later. However, the maximum video resolution of any consumer cameras is at present at $4 \mathrm{~K}$, which may not be the best resolution for objects with minute details.

Time-lapse photography is another option, which provides maximum camera resolution. Time-lapsed photography allows the adjustments of time-based snapshots, usually in seconds. However, the speed of the user walking around the object must be measured accordingly. Time-lapsed photography can be difficult when there is a crowd around your subject. Image clarity can also be an issue, particularly when you have no control over when the shot is taking place. Images taken during movements lose focus.

In our experience, manual shots around your subject are still the best approach. The more photographs captured at different angles of the subject the better. For mass photogrammetry and when time can be a constrain, we propose at least a $10^{\circ}$ angle for each shot around an object, which should yield 60+ images as two cycles are needed - one on the profile elevation and one at a top down view. For objects with detailed surface textures, additional cycles close to the surface are needed. If the object and environment are in perfect conditions, 20 shots for a one-sided capture appears to be sufficient for a medium resolution model, such as the camel in figure 1A. In the case where the bases of objects are important, a third cycle is necessary where shots are taken from a bottom up angle. In general, take as many images as possible. As a guide, professional photogrammetry work uses thousands of photos up to tens of thousands for large, room-sized objects. 


\subsection{Processing and Editing Scans}

Here, we describe our use of Autodesk ReCap as an example for editing models. VisualSFM requires additional processing in other software packages, e.g., MeshLab, for generating polygons from point-clouds. Most software has similar editing tools mentioned in this section. Autodesk ReCap provides a set of easy editing tools such as marquee, lasso, smart brush, face inversion, isolation mode, smartbrush. It also provides mending tools such as smart mesh diagnostics for mending holes and for removing particles, smoothing surfaces, boundaries, as well as a decimate tools.

A collection of multiple angles of an object with overlaps can be uploaded onto Autodesk ReCap. ReCap uses Autodesk Cloud for standard PCs with an Internet connection, VisualSFM and other software packages allows reconstructions locally. The process could take a couple of minutes up to a few hours depending on computing resources and complexity of the images. In the case of ReCap, once the process is completed some editing may be required to remove surrounding environmental artefacts (Top left of Figure 2). This is easy to process using the selection tools to select and remove artefacts, or to simply select the target object and remove the unselected artefacts. The next step is to orientate the model to its appropriate axes. In our experience of processing over 300 heritage objects under difficult environments, more specific editing is necessary. These include mending missing polygons using the 'fill holes' feature, or the 'bridge boundaries' tool to join two edges. Surface tools may be used after filling holes, together with the sculpting and smoothing functions for perfecting the models. There are additional features prior to 3D model export such as the 'detect and fix model issues', which can greatly assist in detecting and fixing issues such as isolated vertices, small gaps and intersects.

Both software have simple features for model editing, professional users may wish to export the model to another package such as 3D Studio Max or Blender 3D to preserve texture maps using 'texture baking', using retopology techniques, and model decimation before export to other platforms (Figure 3). Note that retopologising a 3D object remeshes and replaces it with a user-created mesh, which is essentially an interpreted mesh created by the user. This replaces the original captured model. We have used Adobe Premiere for retrieving time-lapsed photos and short videos into batch JPG images, and Adobe Lightroom for developing RAW images into TIFF files. RAW images capture good information, particularly dark areas which can be adjusted to reduce contrasts.

The figures (Fig. 2 and 3) illustrate different 3D model editing needs for defects and areas not covered by photographs, which our readers could learn from. As the use of software is not our focus in this article, the text contained in this section is sufficient. Each software package has a documented technical guide, which our readers may wish to read. 

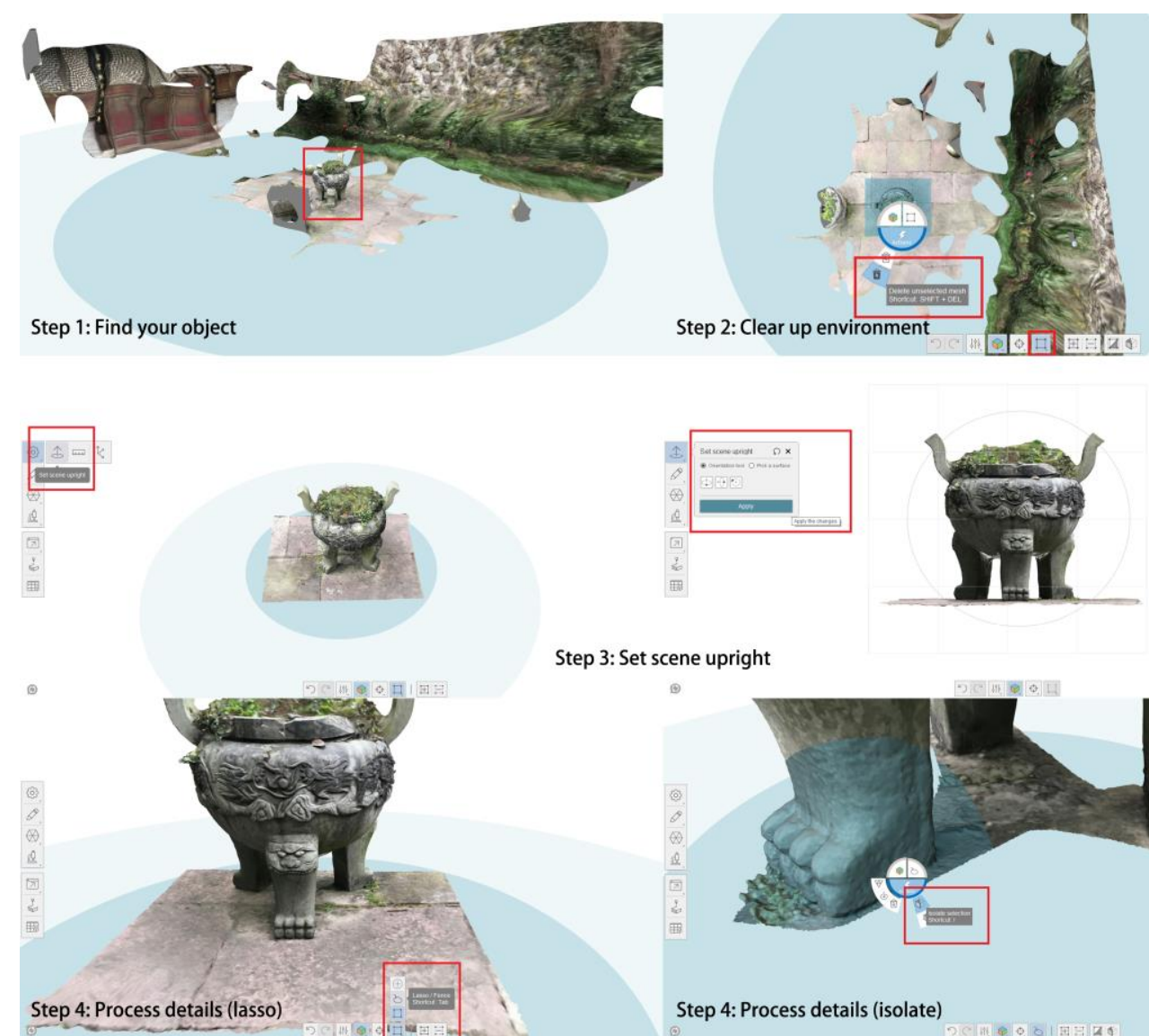

Step 3: Set scene upright

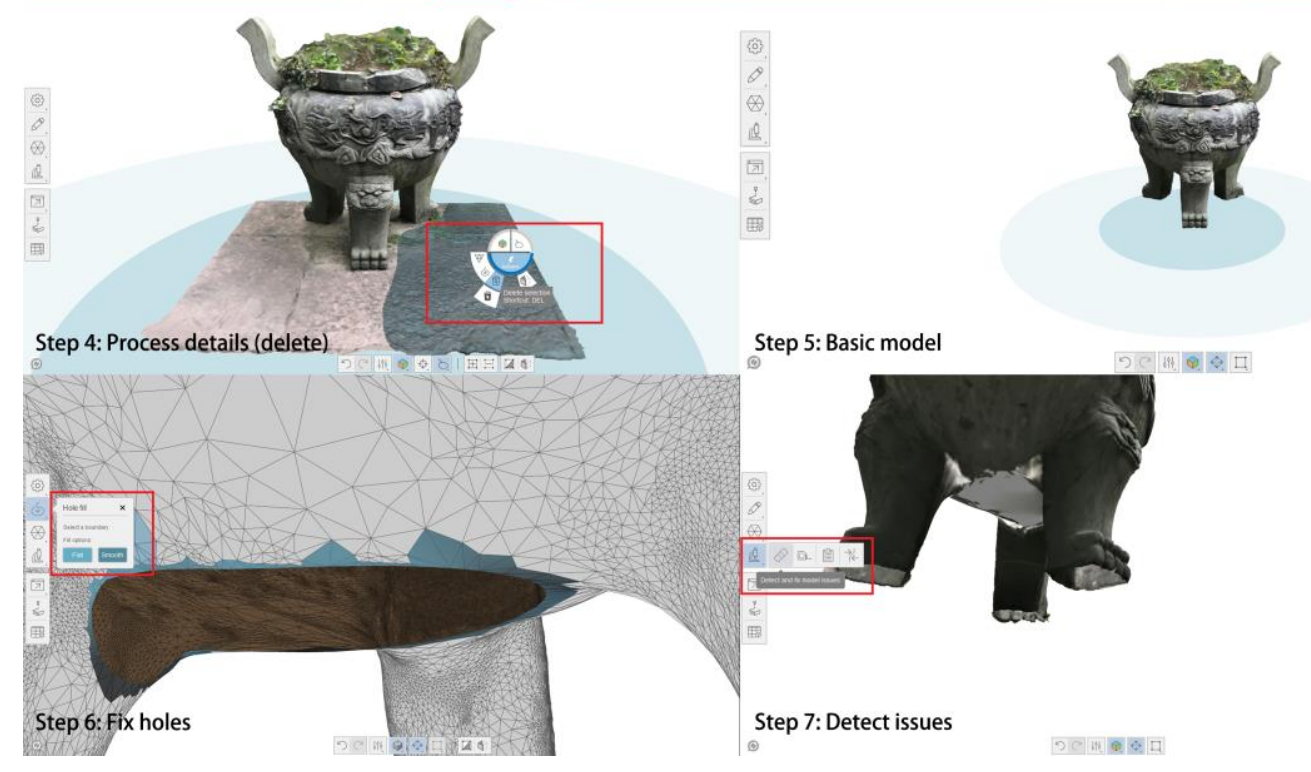

Fig. 2. Processing, filling holes and gaps and editing out defects. 

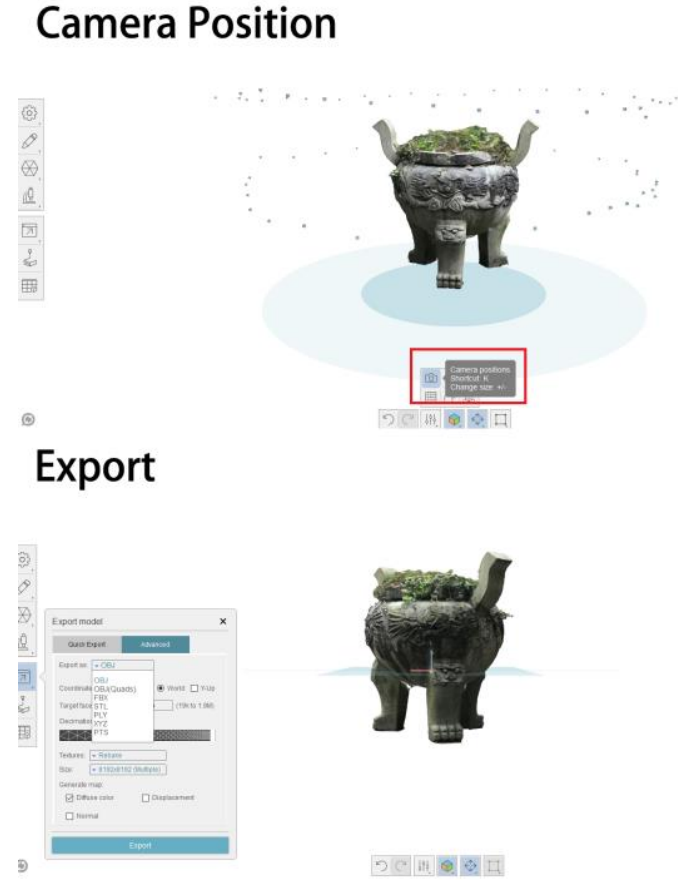

\section{Decimate}

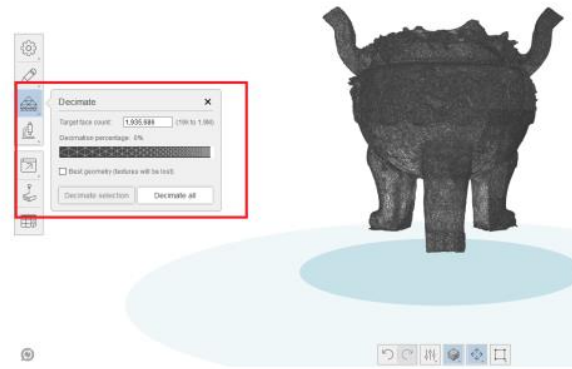

Mesh Report

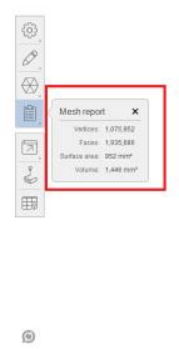

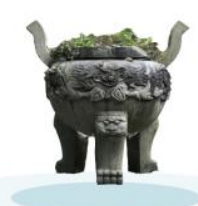

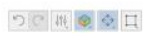

Fig.3. Post-editing a photogrammetry model

\subsection{Exporting for Other Use}

$3 \mathrm{D}$ models at the native level are geometries described by vertices, normal vectors and texture coordinates. Most photogrammetry software has export features supporting numerous formats (e.g., obj, fbx, 3ds, and etc) which are importable by 3D modelling software containing multiple export pipelines for games engines and VR/AR software. Thus, model export is a straightforward process. Autodesk ReCap and VisualSFM allow exports to formats for popular games engines - Unity3D and Unreal Engine. It also exports to the STL 3D printing format. We wish to note that direct exports for detailed models with excessive polygon counts can post challenging problems for games engines.

In our experience, Unity has more issues with importing models than Unreal Engine. Unity automatically segments a model with large polygon counts into chunks of objects grouped as a GameObject, as a Unity scene folder containing multiple chunks of geometries. At the time of writing, the limitation of Unity is that it can only manage 16 bit indices $\left(2^{16}=65536\right)$, meaning that each mesh size is limited to an index buffer in addressing of $64 \mathrm{~K}$ vertices. For Unity, to avoid imported 3D models from being split up into multiple chunks, decimation is necessary. This limit can decrease the original quality of 3D models. However, 3D modelling techniques such as retopology and texture baking will resolve the issue, if not for the 
tediousness of manual retopology. All our models imported without an issue in Unreal Engine.

\subsection{Photogrammetry and Museums}

Photogrammetry work depended mostly on manual labor and other weighted factors as our descriptive formula presented earlier, and described in real-use cases as the methodology has demonstrated. Provided that you do not use camera flash and additional light source, there should be no serious issues. The act of taking many photos from different angles, videorecording by walking around an object, and taking time-lapsed shots do not constitute an offence against policies of museums, unless photography is explicitly prohibited. It is the process behind the scene, at our lab, away from the museum that reconstructs the 3D model. In our experience, security will only intervene when you misbehave by going against the policy of the museums. Some security personnel will inquire, and the best response is to simply explain the purpose of your activity.

\section{Results}

We present the results of our photogrammetry work in this section. We demonstrate the quality results of our simple guideline by presenting models (Figure 6) sampled from our 300 objects within our digital database. We also compared three objects and the effects of photogrammetry between three different devices in section A. Section B presents effects of 3D objects captured in different lighting conditions, $\mathrm{C}$ demonstrates equipment speed comparison between two NVIDIA GPUs. Section D illustrates different 3D model editing needs for defects and areas not covered by photographs.

\subsection{A Comparison of Capturing Devices}

We have mentioned that we compared 3D models captured using three different cameras iPhone 7 Plus, Samsung S7, and Canon EOS 6D Digital SLR. The RAW format in Digital SLRs are particularly useful for capturing information in very dark or very light areas, which can be adjusted later to bring out the surface details in bright/shaded areas. As seen in Figure 4 , there are no significant visual or geometrical differences between devices apart from the colour of the object's texture. 

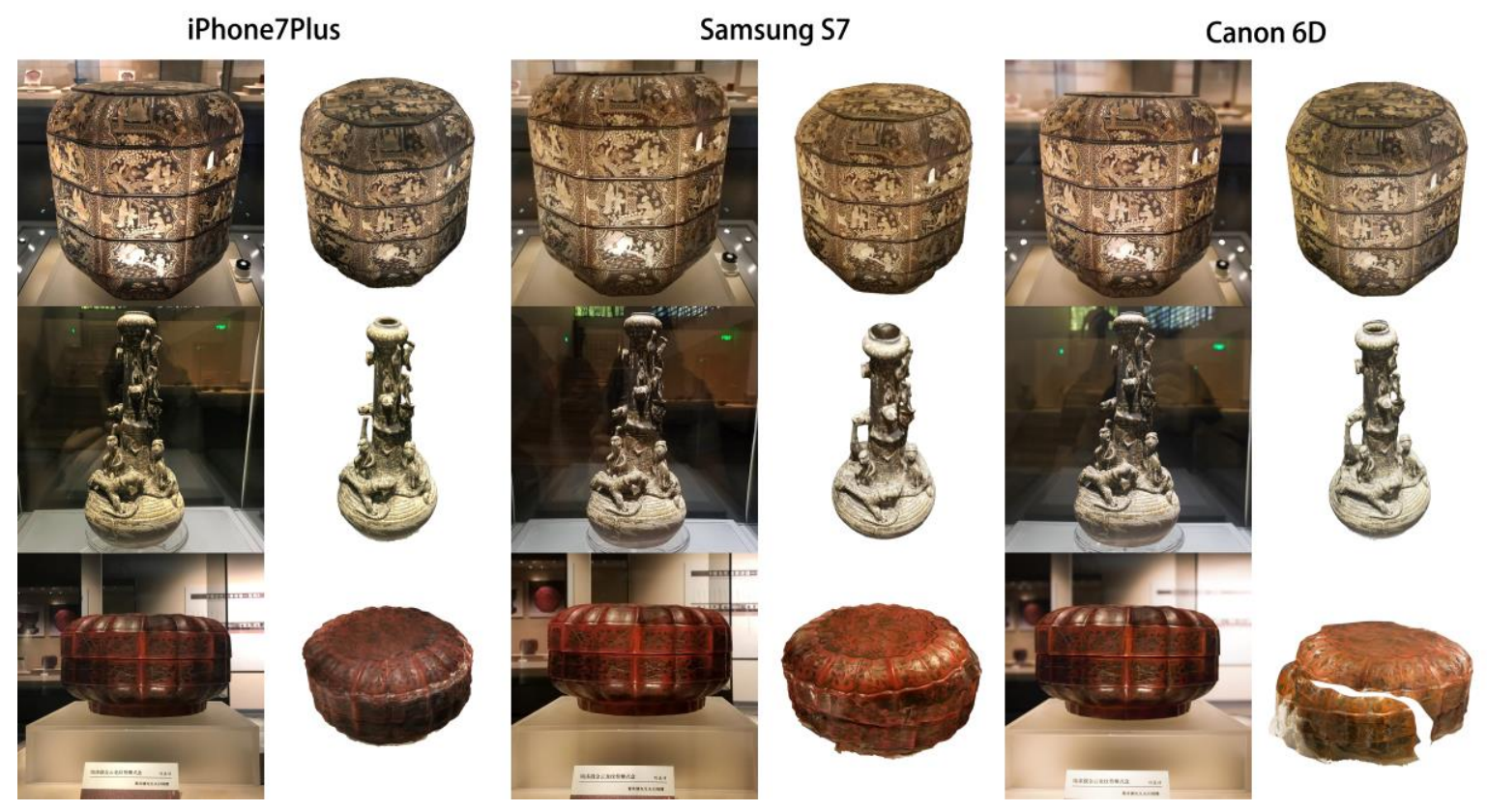

Fig. 4. The figures demonstrate the quality of three different models captured by three different devices.

\subsection{Different Lighting Conditions}

Figure 5 illustrates the effects of lighting conditions on the physical objects and the comparative products of photogrammetry models as a result of different lighting conditions: A) Outdoor strong lighting, B) Outdoor normal lighting, C) indoor normal lighting, D) indoor dark lighting. As a summary, brighter and more even lighting conditions tend to produce better models. Apart from $\mathrm{C}$, which has defects, all other models with moderate to brightly lit environments are of high quality. 

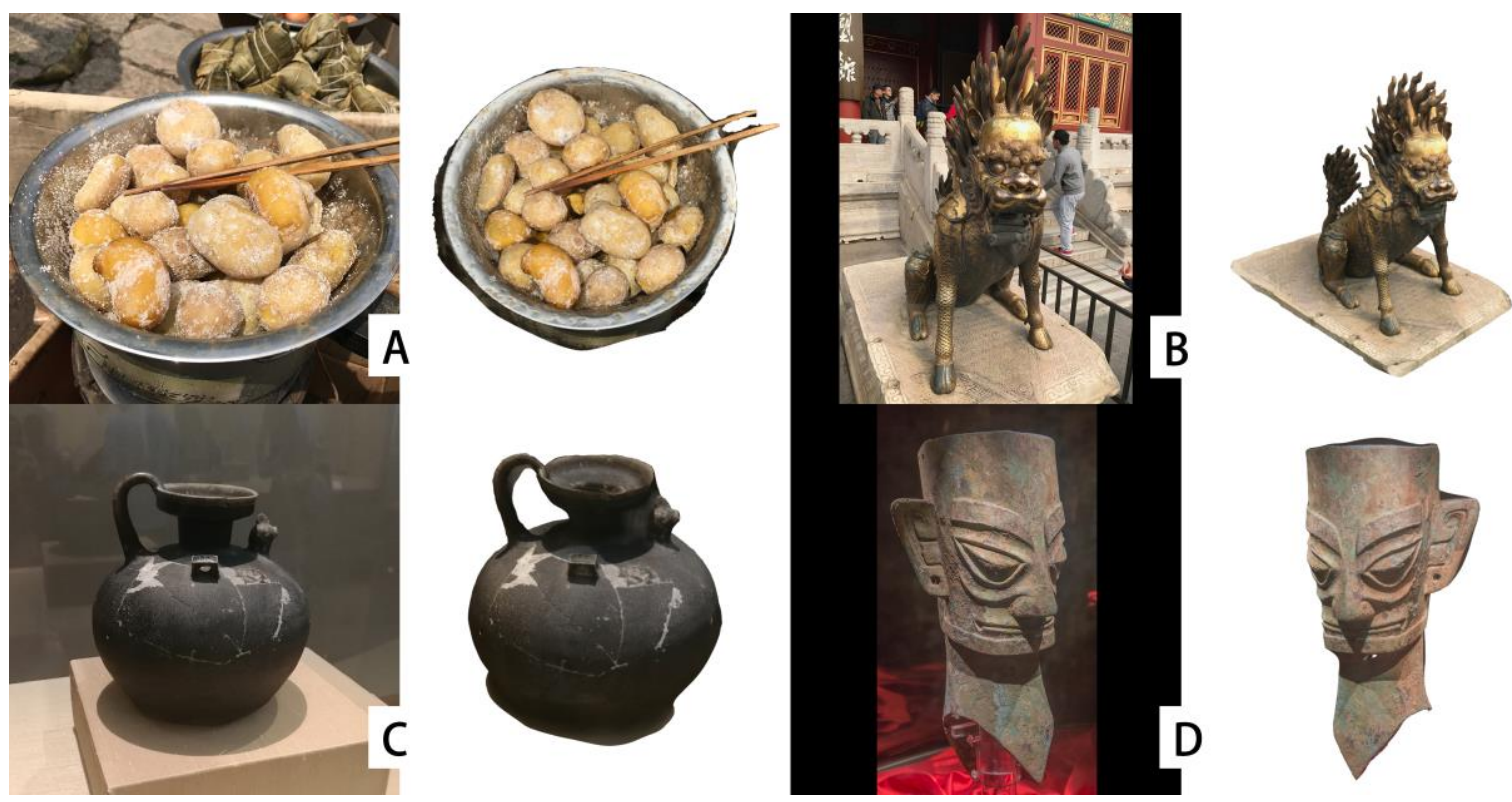

Fig. 5. The figure illustrates the effects of lighting conditions on the physical object, and the product of photogrammetry as a result of the lighting conditions. 


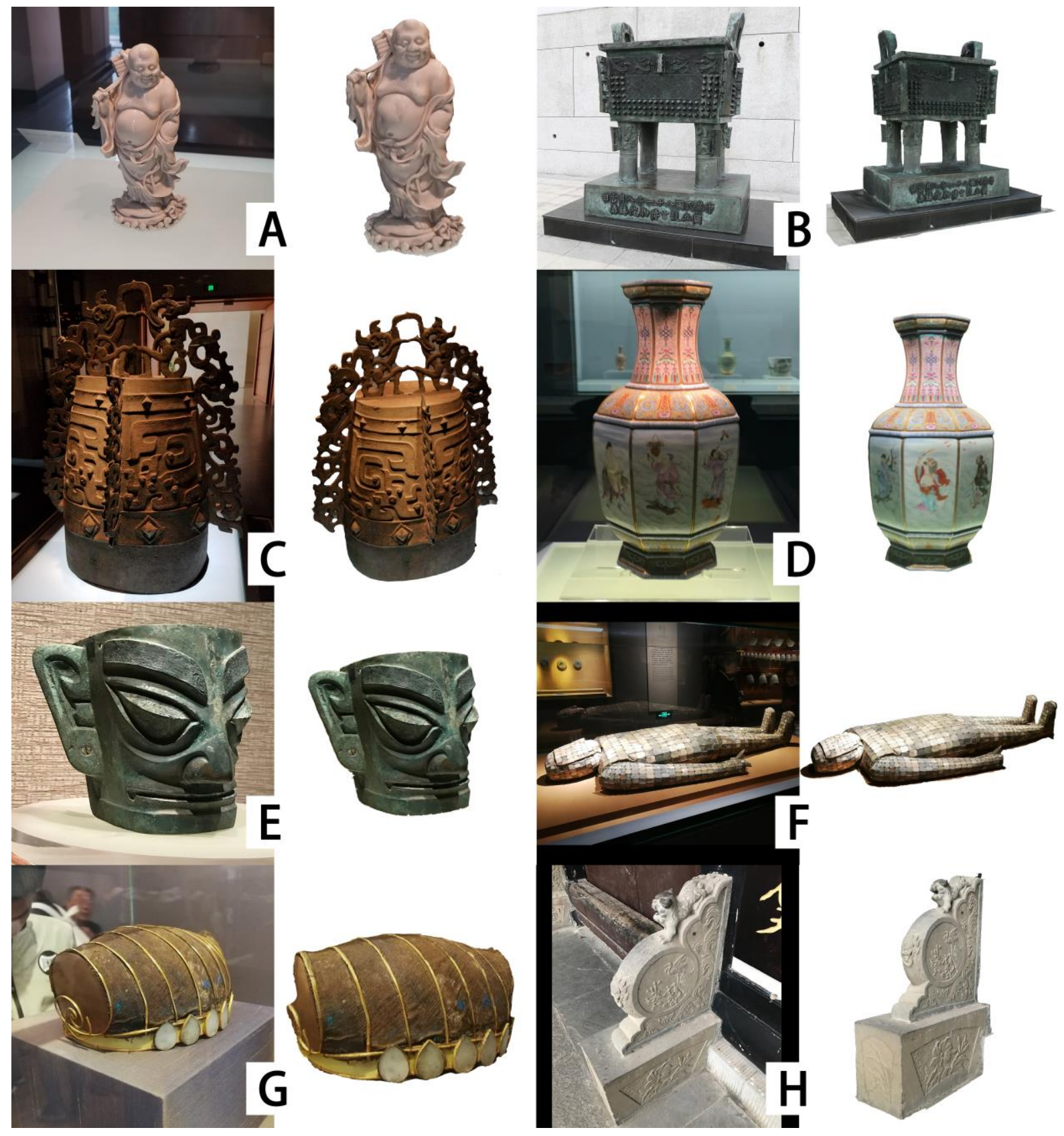

Fig. 6. Samples of photogrammetry reconstructions from our collection of over 300 objects. 


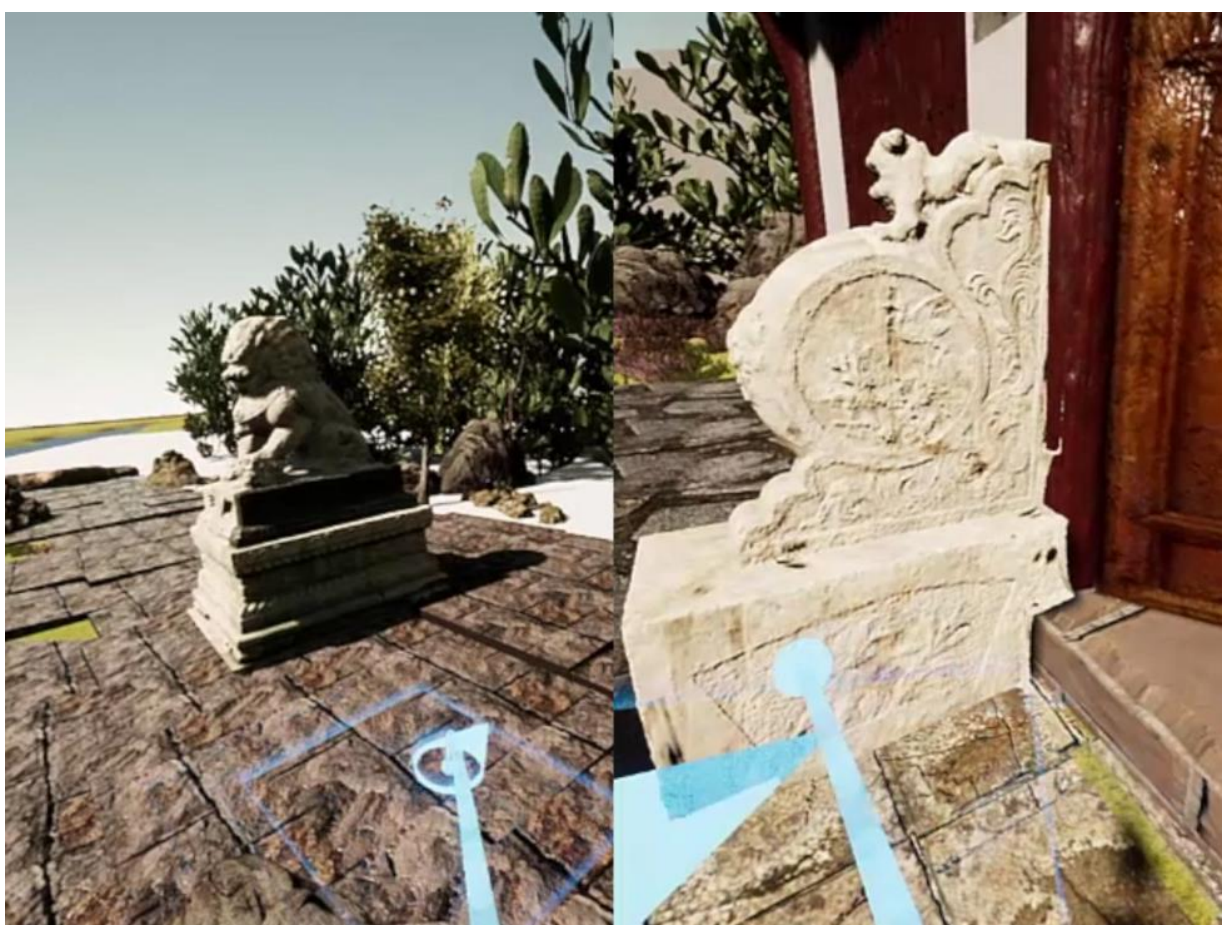

Fig. 7. VR demo with photogrammetry objects. On the left is a large statue of a lion over 7 feet in height and on the right is an ornament decorating a doorway from one of the ancient houses in Beijing's Hutong, in (Leow et al., 2017).

\subsection{GPU Performance Comparisons}

Here, we present reconstruction speed comparisons between two NVIDIA GPUs. We specifically chose a high-end professional card as compared to a basic VR gaming GPU so that our readers may have a way of gauging performance and pricing. Our GPUs are installed in similar motherboards with 32GB of RAM and Intel i7 running Windows 10:

1. NVIDIA Quadro M6000 with 24GB memory is a professional workstation VR GPU with Maxwell architecture. The M6000 has 3840 GPU cores.

2. NVIDIA GEFORCE GTX1080 with 8GB memory is a VR gaming GPU with Pascal architecture. The GTX1080 has 2560 GPU cores.

There are very minor differences in the time needed for the two machines to process and generate 3D models. For the same model, it took 43 minutes 52 seconds for M6000 to process 45 images (123MB in total), and 46 minutes 20 seconds for GTX1080. This again demonstrated that an affordable GPU is all that is needed to generate mass-photography 
quality 3D models. We wish to note in professional photogrammetry work involving thousands of high resolution photographs ( $\sim 50$ megapixels) at $20 \mathrm{MB}$ to $100 \mathrm{MB}$ per file size will require high-end graphics card. As the number of images increases, the high-end cards will yield better performance.

\section{Discussions}

In this article, we articulated the need for the digital recording of cultural heritage via crowdsourcing work at a time when technology has become accessible, and digital transformation within the processes of institutional activities has become mandatory for the reproduction, storage and access of cultural heritage sites, monuments and objects. At the crossroad between technological readiness, global sharing and community culture, government policy changes (see for example policies between the West and the East, Culture is Digital Executive Summary report (Hancock, 2018) and China's Cultural Technology Innovation Plan (Ministry of Culture of the People's Republic of China, 2017), and the lack of resources within cultural institutions such as galleries, libraries, museums and archives, mobilising the masses and integrating large volunteer base within institutional frameworks can be a positive venture. Seeing the need at such a crossroad, we presented the rationale for a guide to mass photogrammetry of cultural heritage with methods refined through the trial and error of documenting over 300 objects across 22 different sites and museums in East Asia. We elaborated that a 'scientific approach' to professional photogrammetry as compared to a 'simplistic approach' used in mass photogrammetry can have very different utility, and that the use-value of professional digital preservation contrasts greatly with the needs in mass photogrammetry. Such a guide covering a gap most needed for mass photogrammetry is promising in that it can assist both amateurs and cultural groups in the practice of digitally recording cultural heritage objects, for learning and sharing, and for creative works. The contents of the guide are extensible for use in GLAMs and other sites hosting creative works such as art and design galleries, retails and etc.

The inclusion of stakeholders is important in sustaining developments, especially when participants and visitors are the core audiences of museum activities. A publication resulting from an ICOMOS conference addresses the need for stakeholder participation in the use of digital technologies for heritage (Lewi and Smith, 2016), promoting participation by allowing "science to be 'developed and enacted by citizens themselves'. We identify the need for both individually-oriented citizen science (Irwin, 2002), and broader community science (Carr, 2004), and see instances where individually oriented citizens carry out works directed by needs, and works specific to more private ventures. Broader communities on the other hand, cover greater ground, acting cohesively for broader public interests befitting that of the society at the time.

We felt that mass photogrammetry will attract widespread attention, the motivation of which can be attributed to an object's cultural identity, which may be valuable in some ways to the persons intending to digitise objects. Activities involving communities have many great benefits, one of which is that they will "open up new ways of exploring and articulating a 
community's relations with the physical and social settings...thereby enabling a form of social production of heritage as the locus of our sense of place." (Giaccardi and Palen, 2008). The issue acquires a more serious tone when the person intends to sell the objects online, or use them as part of a commercial project, such as within a computer game, an edutainment, or a documentary, often without prior permission from the source institution. Derivative works may be created from heritage objects within the creative industry, without crediting the source, with the form of the object, its meaning and identity diluted as a result. By making mass photogrammetry an official activity, and making digitised objects shareable more formally, it will allow cultural institutions to communicate their contents more widely and, as a consequence, inspire creativity in the community. This can be a good reason for cultural institutions to consider the threats posed by mass photogrammetry into IP management opportunities.

Apart from the benefits of digitally recording cultural heritage for learning and sharing, our aim for writing the rationale for a mass photogrammetry guide was for helping cultural institutions in need of a digital team (a digital team differs from general IT departments), but without the financial means to acquire expensive equipment to facilitate their own digital transformation of institutional processes. As stated in an article on China museum's digital heritage profile (White and Ch'ng, to appear 2019), most museums do not have a dedicated digital team capable of interactive 3D and media. With the guide in place, if cultural institutions as hubs for cultural heritage activities can further encourage and make aware of the democratisation of photogrammetry for their own collections, a pool of resources will be made available for education and sharing. Therefore, mobilising visitors for participatory work is greatly encouraged, and is beneficial for cultural institutions lacking human and financial resources. Earlier on, we noted the case whereby the British Museum upon the release of their very first set of 3D models, have since, within three years, scanned only 242 items out of their 8 million on display and in the archives. The opportunity for mass photogrammetry does coincides with the urgent need for citizens to take up voluntary roles in digitally recording cultural heritage which has particular intrinsic and instrumental value for themselves, and for their museums in their communities. Mass photogrammetry practices can be a solution for dwindling interests for heritage for the younger, digital generation, especially when such activities involves digital making and sharing of products, i.e., 3D models via platforms, e.g., SketchFab, which are shareable on social media. Mass photogrammetry is also a first step towards many creative processes using freely available software for narratives and games design, and as a source of creativity and as creative outlets of expression. 150 years ago in 1867, Henry Cole made the prominent people of his age signed The Convention for Promoting Universally Reproductions of Works of Art for the Benefit of Museums of All Countries. The convention promoted the reproduction of art with casts, photographs and electrotypes public education. The ReACH declaration (ReACHDeclaration, 2018), endorsed by UNESCO now promotes digital reproduction of art and cultural heritage for greater benefits to society. Raising public interests for digitising cultural heritage and mobilising them to take part in large-scale digitisation works can have 
impact for local groups and wider society in terms of the creative economy. By providing a means for conducting photogrammetry activities from a crowdsourcing point of view, we believe that citizens globally will be able to record artefacts of both importance and value within cultural institutions, but also heritage artefacts and narratives in the homes and domestic spaces of citizens globally not considered valuable and worthy of record. Private collections that are unseen in the public domain, and as a consequence unstudied can now be made available digitally via 3D model sharing platforms. It is entirely possible that some of these objects may even be missing links in the heritage knowledge base, much like some fossils are bridges to gaps in biological evolution.

Here we wish to restate the purpose of this article, which aims to enhance the social impact of employing low-cost photogrammetry methods for the reproduction of cultural heritage, by allowing the public to help preserve and make accessible local cultural heritage more globally, so that personal cultural heritage objects hidden within the homes of various societies can be presented and thus encouraging cross-border, cross-cultural communications. Such activities will also help to fill information gaps where objects passed down from past generations are unknown to cultural institutions. Secondary to our aim was the issue of IP and copyright raised as a result of mass photogrammetry, which has implications for economic and market-value loss due to unauthorised and widespread use of objects obtained from host institutions without prior permission. Making mass photogrammetry an official crowdsourcing activity, and following the ReACH technical guidelines when reproducing works can resolve such an issue. Whilst beyond the scope of this paper, IP issues raised as a result of 3D 'digital thievery' should become an important awareness for museums in countries where these issues are unmanaged.

\section{References}

Anderson, B. (2006) 'Imagined Communities: reflections on the origin and spread of nationalism'. London: Verso. doi: 10.1080/14725843.2011.556797.

Beyond Productivity: Information Technology, Innovation and Creativity (2003). Washington, D.C: National Academies Press.

Böhler, W. and Marbs, A. (2004) '3D scanning and photogrammetry for heritage recording: a comparison', in Proceedings of the 12th International Conference on Geoinformatics. Gavle University Press, Sweden, pp. 291-298.

Cai, S., Ch'ng, E. and Li, Y. (2018) 'A Comparison of the Capacities of VR and 360-Degree Video for Coordinating Memory in the Experience of Cultural Heritage', in Digital Heritage 2018. San Francisco, USA: IEEE.

Carr, A. J. L. (2004) 'Why do we all need community science?', Society \& Natural Resources.

Ch'ng, E. (2013) 'The Mirror Between Two Worlds: 3D Surface Computing Interaction for Digital Objects and Environments', in Digital Media and Technologies for Virtual Artistic Spaces. Hershey, PA: 
IGI Global. doi: 10.4018/978-1-4666-2961-5.ch013.

Ch'ng, E., Gaffney, V. L. and Chapman, H. P. (2014) 'From Product to Process: New Directions in Digital Heritage', in Din, H. and Wu, S. (eds) Digital Heritage and Culture : Strategy and Implementation. 1st edn. World Scientific, pp. 219-243.

Derclaye, E. (2010) Copyright and Cultural Heritage: Preservation and access to works in a digital world. Edward Elgar Publishing.

Giaccardi, E. and Palen, L. (2008) 'The social production of heritage through cross-media interaction: making place for place-making', International Journal of Heritage Studies. Taylor \& Francis, 14(3), pp. 281-297.

Granshaw, S. I. (2015) 'Imaging Technology 1430-2015: Old Masters to Mass Photogrammetry', Photogrammetric Record, 30(151), pp. 255-260. doi: 10.1111/phor.12112.

Greffe, X. (2004) 'Is heritage an asset or a liability?', Journal of Cultural Heritage, 5(2004), pp. 301309.

Hanan, H., Suwardhi, D., Nurhasanah, T. and Santa Bukit, E. (2015) 'Batak Toba cultural heritage and close-range photogrammetry', Procedia-Social and Behavioral Sciences. Elsevier, 184, pp. 187-195.

Hancock, M. (2018) 'Culture is Digital: Executive summary'. Department for Digital, Culture, Media and Sport. Available at: https://www.gov.uk/government/publications/culture-is-digital/culture-isdigital.

Hughes, R. (2015) 'The International Council of Monuments and Sites: A New Digital Technology National Committee', ICOMOS International Council on Monuments and Sites UK, II.

Irwin, A. (2002) Citizen science: A study of people, expertise and sustainable development. Routledge.

Koller, D. and Levoy, M. (2004) 'Computer-Aided Reconstruction and New Matches in the Forma Urbis Romae', in Formae Urbis Romae - Nuove scoperte.

Leow, F.-T., Ch'ng, E., Zhang, T., Cai, S. and See, S. (2017) “'In-The-Wild” Observation and Evaluation of a Chinese Heritage VR Environment with the HTC VIVE', in International Conference on Virtual Systems and Multimedia (VSMM), 31 Oct - 2 Nov. Dublin, Ireland.

Lewi, H. and Smith, W. (2016) 'Citizen Heritage: Provoking participation in place through digital technologies', Historic Environment. Council for the Historic Environment, Australia, 28(2), p. 2.

Li, Y., Ch'ng, E., Cai, S. and See, S. (2018) 'Multiuser Interaction with Hybrid VR and AR for Cultural Heritage Objects', in Digital Heritage 2018. San Francisco, USA: IEEE. Available at:

file:///Users/yueli/Library/Application Support/Mendeley Desktop/Downloaded/Li et al. - 2018 Multiuser Interaction with Hybrid VR and AR for Cultural Heritage Objects.pdf.

Luhmann, T., Robson, S., Kyle, S. A. and Harley, I. A. (2006) Close range photogrammetry: principles, techniques and applications. Whittles. 
Margoni, T. (2014) 'The digitisation of cultural heritage: originality, derivative works and (non) original photographs'.

Miles, H. C., Wilson, A. T., Labrosse, F., Tiddeman, B., Griffiths, S., Edwards, B., Ritsos, P. D., Mearman, J. W., Möller, K. and Karl, R. (2016) 'Alternative representations of 3D-reconstructed heritage data', Journal on Computing and Cultural Heritage (JOCCH). ACM, 9(1), p. 4.

Ministry of Culture of the People's Republic of China (2017) Cultural Technology Innovation Plan during 13th Five Year period from Ministry of Culture (文化部“十三五”时期文化科技创新规划).

Nightingale, V. (2007) 'The cameraphone and online image sharing', Continuum: Journal of media \& cultural studies. Taylor \& Francis, 21(2), pp. 289-301.

Noordegraaf, J., Bartholomew, A. and Eveleigh, A. (2014) 'Modeling Crowdsourcing for Cultural Heritage', in MW2014: Museums and the Web 2014.

Pantalony, R. E. (2006) WIPO guide on managing intellectual property for museums. WIPO.

Potts, J. and Cunningham, S. (2008) 'Four models of the creative industries', International Journal of Cultural Policy, 14(3), pp. 233-247. doi: 10.3917/redp.201.0163.

Project, R. (2016) Digital heritage: intellectual rights, democracy and commoditisation of cultural heritage places, RICHES Think Paper Collection.

ReACHDeclaration (2018) 'ReACH: Reproduction of Art and Cultural Heritage', Victoria and Albert Museum Publishing. Available at: https://www.vam.ac.uk/research/projects/reach-reproduction-ofart-and-cultural-heritage\#outputs.

Remondino, F. (2011) 'Heritage recording and 3D modeling with photogrammetry and 3D scanning', Remote Sensing. Molecular Diversity Preservation International, 3(6), pp. 1104-1138.

Rocchini, C., Cignoni, P., Montani, C., Pingi, P. and Scopigno, R. (2001) 'A low cost 3D scanner based on structured light', in Computer Graphics Forum. Wiley Online Library, pp. 299-308.

Sabiescu, A., Woolley, M., Cummings, C. and Prins, J. (2015) 'Online Maker Communities: Craft and Engagement with Cultural Heritage', in Conference Communities and Technologies, Limerick, Ireland.

Samaan, M., Deseilligny, M. P., Heno, R., Vaissière, E. D. La and Roger, J. (2016) 'Close-Range Photogrammetric Tools for Epigraphic Surveys', Journal on Computing and Cultural Heritage (JOCCH). ACM, 9(3), p. 16.

Sansoni, G., Trebeschi, M. and Docchio, F. (2009) 'State-of-the-art and applications of 3D imaging sensors in industry, cultural heritage, medicine, and criminal investigation', Sensors. Molecular Diversity Preservation International, 9(1), pp. 568-601.

Shirky, C. (2010) Cognitive surplus: Creativity and generosity in a connected age. Penguin UK. Swearingen, S. and Swearingen, K. L. (2016) 'Creating virtual environments with 3D printing and 
photogrammetry', in SIGGRAPH ASIA 2016 Creating Virtual Environments. ACM, p. 1.

Tanenbaum, J. G., Williams, A. M., Desjardins, A. and Tanenbaum, K. (2013) 'Democratizing technology: pleasure, utility and expressiveness in DIY and maker practice', Proceedings of the SIGCHI Conference on Human Factors in Computing Systems, pp. 2603-2612. doi: $10.1145 / 2470654.2481360$.

Thurston, A. (2012) Digital preservation: preserving heritage and protecting civil rights, UNESCO Press.

TNN (2017) Heritage Enthusiasts Seek to Crowdsource Global Search for Stolen Indian Treasures.

White, A. and Ch'ng, E. (no date) 'China Museum's Digital Heritage Profile: An Evaluation of Digital Technology Adoption in Cultural Heritage Institutions', in Cooke, S., Lewi, H., and Smith Wally (eds) DigitalGLAM interrogating the creative development of new digital media in the cultural sector. Routledge Museum Studies Series.

Wu, C. (2011) 'VisualSFM: A visual structure from motion system'.

Yastikli, N. (2007) 'Documentation of cultural heritage using digital photogrammetry and laser scanning', Journal of Cultural Heritage. Elsevier, 8(4), pp. 423-427.

Younan, S. (2015) 'Poaching Museum Collections using Digital 3D Technologies', Journal of Science and Technology of the Arts, 7(2), pp. 25-32.

Zarndt, F., Geiger, B., Pacy, A. and Boddie, S. (2013) 'Crowdsourcing the World's Cultural Heritage, Part II', in 2013 IFLA World Library and Information Congress - Singapore. 